\title{
Genetic marker polymorphisms on chromosome 8q24 and prostate cancer in the Dutch population: DG8S737 may not be the causative variant
}

\author{
Maurice P Zeegers ${ }^{1,2,8}$, Humera S Khan ${ }^{\star 1,8}$, Leo J Schouten ${ }^{3}$, Boukje AC van Dijk ${ }^{4}, \mathrm{R}$ Alexandra Goldbohm ${ }^{5}$, \\ Jack Schalken ${ }^{6}$, Shahin Shajahan ${ }^{7}$, Alexander Pearlman ${ }^{7}$, Carole Oddoux ${ }^{7}$, Piet A van den Brandt ${ }^{3}$ and \\ Harry Ostrer ${ }^{7}$
}

Prostate cancer is the most commonly diagnosed cancer in men in Europe and Northern America. Genome-wide association studies (GWAS) have detected an association with markers on chromosome 8q24. Allele -8 of microsatellite DG8S737 with 22 repeats and allele $A$ of the single-nucleotide polymorphism (SNP) rs 1447295 have been found to be significantly associated with prostate cancer. As GWAS are subjected to type 1 error, confirmation studies are required to validate the results. Here, we analysed the same markers in $\mathbf{2 7 7}$ cases and $\mathbf{2 8 2}$ controls from the Netherlands using a nested case-control study. Incident prostate cancer cases and controls selected were identified in the population of the Netherlands Cohort Study. We also investigated clinical features of the disease by stratifying by tumour stage. We did not replicate the association with the SNP rs1447295-A allele $(P=0.10)$, although the effect estimate was in the same direction as previous studies (odds ratio (OR), 1.38). Interestingly a statistically significant decreased risk was observed for DG8S737 allele -8 (OR, $0.62 ; P=0.03$ ). The apparent protective effect of the DG8S737 -8 allele observed in this study contrasts with the Amundadottir study. This suggests that DG8S737 and rs1447295 might be tightly linked markers flanking the actual causative variant and that there may be potentially more than one high-risk haplotype present in the Caucasian population. This short report highlights the importance of validation, although further confirmation is still needed.

European Journal of Human Genetics (2011) 19, 118-120; doi:10.1038/ejhg.2010.133; published online 11 August 2010

Keywords: epidemiology; microsatellite repeats/genetics; SNP; prostatic neoplasm/genetics; cancer

\section{INTRODUCTION}

The research by the Surveillance, Epidemiology and End Results (SEER) Program of the National Cancer Institute estimated prostate cancer to be the most common cancer diagnosis in men in Europe and the United States of America. It was estimated that $\sim 192280$ new cases would arise in 2009, representing $25 \%$ of all new cases of cancer among men. ${ }^{1}$ In 2006, a genome-wide linkage analysis of 877 Icelandic men with prostate cancer demonstrated a positive signal on chromosome 8q24 (logarithm of odds score: 2.11). The variant allele -8 (22 repeats) of the microsatellite DG8S737 had the strongest signal in a follow-up association study (odds ratio (OR), 1.79; $P=3.0 \times 10^{-6}$ ). This was replicated in Icelandic men $\left(\mathrm{OR}, 1.72 ; P=1.8 \times 10^{-3}\right)$ and among Swedish and European American men. In addition, allele A of the rs1447295 single-nucleotide polymorphism (SNP) present within the same haplotype block was also found to have a significant association with prostate cancer (OR, 1.72; $\left.P=1.7 \times 10^{-9}\right)$. Analysis of 598 African-American men confirmed the results for DG8S737, but not for rs1447295, consistent with the greater genetic diversity of this population. ${ }^{2}$ Another recent replication study confirmed the association of rs1447295-A allele with prostate cancer in European men aged 40-64 years from Washington, but not the association with allele -8 of DG8S737. ${ }^{3}$ Both the rs1447295-A allele and DG8S737-10 allele were significantly associated with high grade tumours (OR, 1.4; 1.1-1.8 and OR, $1.9 ; 1.2-2.8$, respectively). ${ }^{3}$ Two recently reported genome-wide association studies (GWAS) confirmed the association of the rs1447295-A allele and prostate cancer risk, ${ }^{4,5}$ but did not stratify by tumour stage. The variability in haplotype association results may be related to tumour stage heterogeneity or genetic heterogeneity within and between populations.

We have previously investigated the founder mutations among the Dutch and found mutations that were specific for this population, including those predisposing for hereditary breast-ovarian cancer and malignant melanoma. ${ }^{6}$ We also identified short chromosomal regions that have remained identical by descent resulting in relatively limited genetic heterogeneity within this population ${ }^{6}$ thus increasing the power in detecting the associations among the Dutch. GWAS often experience type 1 errors, and therefore confirmation of results are required. Here, we tested whether the association between rs1447295

\footnotetext{
${ }^{1}$ Unit of Urologic and Genetic Epidemiology, Department of Public Health, Epidemiology \& Biostatistics, The University of Birmingham, Birmingham, UK; ${ }^{2}$ Department of Complex Genetics, Cluster of Genetics and Cell Biology, Nutrition and Toxicology Institute, Maastricht University, Maastricht, The Netherlands; ${ }^{3}$ Department of Epidemiology, School for Oncology and Developmental Biology (GROW), Maastricht University, Maastricht, The Netherlands; ${ }^{4}$ Comprehensive Cancer Centre North East, Groningen, The Netherlands; ${ }^{5}$ Department of Prevention and Health, TNO Quality of Life, Business Unit Prevention and Care, Leiden, The Netherlands; ${ }^{6}$ Department of Urology, Radboud University Nijmegen Medical Center, Nijmegen, The Netherlands; ${ }^{7}$ Human Genetics Program, Department of Medicine, School of Medicine, New York University, New York, NY, USA

${ }^{*}$ Correspondence: HS Khan, Unit of Urologic and Genetic Epidemiology, Department of Public Health, Epidemiology and Biostatistics, University of Birmingham, Public Health Building, Birmingham, B15 2TT, UK. Tel: +44 121414 7866, Fax: +44 121414 2528; E-mail: a.h.khan@bham.ac.uk

${ }^{8}$ These authors contributed equally to this work.

Received 4 February 2010; revised 21 June 2010; accepted 24 June 2010; published online 11 August 2010
} 
Table 1 Crude and multivariable adjusted odds ratios for rs1447295 and DG8S737

\begin{tabular}{|c|c|c|c|c|c|c|c|}
\hline & Alleles & Cases (n) & Controls (n) & \multicolumn{2}{|c|}{ Unadjusted } & \multicolumn{2}{|c|}{ Adjusted $^{\mathrm{a}}$} \\
\hline \multicolumn{8}{|l|}{$r s 1447295$} \\
\hline Allelic association & Allele A present & 142 & 114 & $1.38(0.94-2.02)$ & 0.1 & $1.39(0.94-2.04)$ & 0.1 \\
\hline Genotypic association & $\mathrm{C} / \mathrm{C}$ & 224 & 196 & 1 & & 1 & \\
\hline \multicolumn{8}{|l|}{ DG8S737 } \\
\hline \multirow[t]{3}{*}{ Allelic association } & Allele -8 absent & 417 & 394 & 1 & & 1 & \\
\hline & Allele -8 present & 43 & 66 & $0.61(0.39-0.94)$ & 0.03 & $0.62(0.40-0.96)$ & 0.03 \\
\hline & Allele -10 absent & 417 & 430 & 1 & & 1 & \\
\hline
\end{tabular}

adjusted for age, alcohol intake from wine, body mass index, energy intake, family history of prostate cancer and level of education.

and DG8S737 and prostate cancer can be confirmed among a population-based Dutch sample and whether this association is different for localised versus advanced tumours.

\section{METHODS}

\section{Study population}

Incident prostate cancer cases and controls were identified in the population of the Netherlands Cohort Study (NLCS); further details can be found elsewhere. ${ }^{7}$ In brief, the NLCS includes 58279 men who were between the ages 55 and 69 years at baseline. This paper reports from the data set after 8.3 years of follow-up. A case-cohort approach was used. Cases were identified after follow-up using computerised record linkage with all nine cancer registries in the Netherlands. Controls were selected from a random subcohort sample of 2411 men and biennially followed up for information on vital status.

\section{Biological samples}

The NLCS cohort was linked to the Dutch pathology database to collect paraffin blocks of tumour and normal tissue samples of prostate cancer cases. After exclusion of prevalent cases and those with insufficient non-tumourous tissue, 300 cases were available for analysis. We obtained buccal swab samples from 300 controls from the NLCS subcohort. The DG8S737 microsatellite marker ( $169 \mathrm{bp}, 128.433 \mathrm{loc}$ ) was amplified using 5'-TGATGCACCACAGAAA CCTG- $3^{\prime}$ as a forward primer and $5^{\prime}$-CAAGGATGCAGCTCACAACA- $3^{\prime}$ as a reverse primer. PCRs were set up using a HYDRA workstation (Matrix Technologies Corporation, Hudson, NH, USA) and run on MJR Thermocyclers (MJ Research, Incorporated, Waltham, MA, USA). The PCR products of cases and subcohort controls were sized on the Applied Biosystems 3730 Sequencer in comparison with CEPH1347-02 reference specimen. Genotyping was performed using Genemapper software version 4.0 (Applied Biosystems, Foster City, CA, USA). The Taqman SNP Genotyping Assay (Applied Biosystems) was used for allele typing of the rs1447295 SNP marker with an ABI PRISM 7900HT (Applied Biosystems).

\section{Statistical analysis}

Deviation from Hardy-Weinberg equilibrium for the genotypes of each marker and linkage disequilibrium between marker alleles was tested by $\chi^{2}$-tests. We calculated ORs and corresponding 95\% confidence intervals (95\% CIs) using logistic regression for allelic and genotypic analysis of the SNP and microsatellite. For allelic analyses, robust SEs have been calculated to model potential clustering of alleles within individuals. In microsatellite analyses, alleles -8 and -10 were compared with all other alleles. The microsatellite was further tested at different breakpoints to test association between groups of alleles. We also tested the marker using allele - 14 (most common allele) as the reference group. Both crude and multivariable (age, alcohol intake from wine, body mass index, energy intake, family history of prostate cancer and level of education) adjusted analyses were performed. We evaluated differences in associations with the localised tumour stage (T0-2, M0) or advanced (T3-4, M0 and T0-4, M1). Stage was reported by the cancer registries and coded according to the International Union Against Cancer tumour-node-metastasis (UICC TNM). ${ }^{8}$ All statistical analyses were performed using Stata 9.0 (StataCorp, 2005; Stata Statistical Software: Release 9, StataCorp LP, College station, TX, USA).

\section{RESULTS}

After excluding subjects with missing data, we were left with 277 cases and 282 controls. These did not significantly differ from their respective case and control source populations with regard to age $\left(P_{\text {cases }}=0.50, P_{\text {controls }}=0.50\right)$ and family history of prostate cancer $\left(P_{\text {cases }}=0.95, P_{\text {controls }}=0.38\right)$. The mean age at baseline of cases in the study population was 63 years, which was significantly higher than the mean age of the subcohort (60 years). A small proportion of the combined sample had positive family history of prostate cancer (4.13\%). The frequency of alleles and genotypes for the microsatellite marker DG8S737 and the SNP rs1447295 did not deviate from Hardy-Weinberg equilibrium among the controls $(P=0.14)$. There was strong evidence for linkage disequilibrium between allele -10 of DG8S737 and the A allele of the SNP $\left(\chi^{2}=61.2, P<0.001\right)$. Table 1 shows the results of the microsatellite and SNP analyses. DG8S737 marker allele -8 (22 repeats) was unexpectedly associated with a statistically significant decreased risk for prostate cancer (OR, 0.62; 95\% CI, $0.40-0.96 ; P=0.03$ ). The increased frequency of the -10 allele among cases was suggestive of the association with increased risk of prostate cancer; however, it did not achieve statistical significance (OR, 1.50; 95\% CI, 0.88-2.55). The results of all alleles can be found in Supplementary Table 1. The SNP analysis suggested an increase in the crude OR with the presence of allele A, (OR, 1.38; 95\% CI, 0.94$2.20)$, although not statistically significant $(P=0.10)$. This was also true for carriers of one and two copies of allele A (OR, 1.40; $P=0.14$ and $\mathrm{OR}, 1.54 ; P=0.48$, respectively). After multivariable adjustment, the results did not differ substantially, suggesting that an assumption of Mendelian randomisation can be made in this context. ${ }^{9}$ No influence was observed for TNM stage (data not shown). 


\section{DISCUSSION}

A novel result of our study was a statistically significant decreased risk associated with allele -8 of the microsatellite marker (OR, 0.62; $95 \%$ CI, $0.40-0.96, P=0.03$ ). This is unlikely to be a result of population structure, given the matching of cases and controls, the absence of apparent outliers or mismatching of ancestry observed by Structure analysis of the Codis set of microsatellite markers and the relative genetic homogeneity of the Dutch population. ${ }^{10,11}$ The apparent protective effect of the DG8S737 -8 allele observed in this study contrasts with the study by Amundadottir study, ${ }^{2}$ which detected an increased risk associated with this allele in Icelandic and Swedish men. Their observation of an association of the -8 allele in conjunction with the A allele of rs1447295 in all of the Caucasian populations studied, but not in the more genetically diverse African-American population, led them to conclude that the variant responsible for conferring the increased prostate cancer risk must be the DG8S737 -8 allele itself or be extremely close to it. The results of this study and those of the Suuriniemi study, ${ }^{3}$ which detected no association with prostate cancer risk for this allele in Caucasian men from Washington, but instead found an association with the -10 allele after stratifying by stage of disease (OR, 1.93; 95\% CI, 1.27-2.93), suggest that this allele itself is not directly responsible for conferring increased prostate cancer risk. It is more likely that DG8S737 and rs1447295 are tightly linked markers flanking the actual causative variant and that there may be potentially more than one high-risk haplotype present in the Caucasian population. This hypothesis might also explain the observations of the Mayo Clinic study that demonstrated that the 8/A haplotype showed the strongest association with familial prostate cancer, whereas the 10/A haplotype was most strongly associated with aggressive prostate cancer. ${ }^{12}$ Comparison of detailed haplotypes in this locus among populations may serve as useful means of further narrowing this interval and assisting with identification of the causative variant.

\section{CONFLICT OF INTEREST}

The authors declare no conflict of interest.

\section{ACKNOWLEDGEMENTS}

This study was established with financial support from the Dutch Cancer Society and the US Department of Defense.

1 Jemal A, Siegel R, Ward E, Hao Y, Xu J, Thun MJ: Cancer statistics, 2009. CA Cancer J Clin 2009; 59: 225-249.

2 Amundadottir LT, Sulem P, Gudmundsson J et al: A common variant associated with prostate cancer in European and African populations. Nat Genet 2006; 38: 652-658.

3 Suuriniemi M, Agalliu I, Schaid DJ et al: Confirmation of a positive association between prostate cancer risk and a locus at chromosome 8q24. Cancer Epidemiol Biomarkers Prev 2007; 16: 809-814.

4 Yeager M, Orr N, Hayes RB et al: Genome-wide association study of prostate cancer identifies a second risk locus at 8q24. Nat Genet 2007; 39: 645-649.

5 Gudmundsson J, Sulem P, Manolescu A et al: Genome-wide association study identifies a second prostate cancer susceptibility variant at 8q24. Nat Genet 2007; 39: 631-637.

6 Zeegers MP, van Poppel F, Vlietinck R, Spruijt L, Ostrer H: Founder mutations among the Dutch. Eur J Hum Genet 2004; 12: 591-600.

7 van den Brandt PA, Goldbohm RA, van 't Veer P, Volovics A, Hermus RJ, Sturmans F: A large-scale prospective cohort study on diet and cancer in the Netherlands. J Clin Epidemiol 1990; 43: 285-295.

8 Sobin LH, Fleming ID: TNM Classification of Malignant Tumors, fifth edition (1997). Union Internationale Contre le Cancer and the American Joint Committee on Cancer. Cancer 1997; 80: 1803-1804.

9 Ebrahim S, Davey Smith G: Mendelian randomization: can genetic epidemiology help redress the failures of observational epidemiology? Hum Genet 2008; 123: 15-33.

10 Sarkar NKV: Genetic diversity at two pentanucleotide STR and thirteen tetranucleotide STR loci by multiplex PCR in four predominant population groups of central India. Forensic Science International 2002; 128: 196-201.

11 Pritchard JK SM, Stephens M, Donnelly P: Inference of population structure using multilocus genotype data. Genetics 2000; 155: 945-959.

12 Wang L, McDonnell SK, Slusser JP et al: Two common chromosome 8q24 variants are associated with increased risk for prostate cancer. Cancer Res 2007; 67: 2944-2950.

Supplementary Information accompanies the paper on European Journal of Human Genetics website (http://www.nature.com/ejhg) 